\title{
Optimal indicator for histogram analysis of fractional anisotropy for normal-appearing white matter in multiple sclerosis
}

\author{
${ }^{1,2}$ Kimihiro Ogisu $P h D,{ }^{3}$ Masaaki Niino $M D P h D,{ }^{3,4}$ Yusei Miyazaki $M D P h D,{ }^{4}$ Seiji Kikuchi \\ $M D P h D$ \\ ${ }^{1}$ Department of Radiology, National Hospital Organization Hokkaido Medical Center, Sapporo; \\ ${ }^{2}$ Department of Radiology, National Hospital Organization Hakodate Hospital, Hakodate; ${ }^{3}$ Department \\ of Clinical Research, ${ }^{4}$ Department of Neurology, National Hospital Organization Hokkaido Medical \\ Center, Sapporo, Japan
}

\begin{abstract}
Background: Normal-appearing white matter (NAWM) lesions are known to be present in multiple sclerosis (MS); however, it is not easy to distinguish these lesions from others in MRI. This study aimed to investigate the most useful value for estimating NAWM damage using fractional anisotropy (FA) histograms analysis. Methods: Data from patients with relapsing-remitting MS and healthy controls were analyzed using a 1.5T MRI system with SENSE-Head-8 coil. FA maps with diffusionweighted images were acquired using a single-shot echo-planar imaging sequence. The median, standard deviation (SD), kurtosis, and skewness of white matter (WM) of each subject were compared between MS and healthy controls using an in-house application. Results: FA decrease in 8 patients with MS was observed upon comparison with 12 controls and leaned toward the left side. While the SDs of the healthy controls were not significantly different from those of patients with MS, patients with MS expressed significantly lower median values, and higher kurtosis and skewness compared to healthy controls. A trend for inverse associations existed between median and expanded disability status scale scores.

Conclusion: Our data suggests that median FA values can allow for distinguishing between patients with MS and healthy controls with high accuracy.
\end{abstract}

Keywords: Multiple sclerosis, magnetic resonance imaging, white matter, expanded disability status scale

\section{INTRODUCTION}

Multiple sclerosis (MS) is a chronic inflammatory disorder of the central nervous system characterized by the presence of demyelinated lesions and axonal loss. ${ }^{1-3}$ The presence of white matter (WM) lesions in the brain is one of the radiological features of MS. ${ }^{4}$ Conventional magnetic resonance imaging (MRI) techniques including T2- and T1-weighted images as well as fluid attenuated inversion recovery (FLAIR) are sensitive for the detection of MS lesions. ${ }^{5,6}$ However, this technology is limited due to a lack of pathologic specificity and a poor correlation with disability. ${ }^{7}$

Diffusion tensor imaging (DTI) is an MRI scanning technique used to characterize the microstructural organization of brain tissue so that measurement of the nuclear magnetic resonance signal is accompanied by diffusion sensitization in multiple directions. ${ }^{8} \mathrm{WM}$ is a well-organized and densely packed fiber structure. WM fiber tracts consist of aligned myelinated axons that play a role as a barrier; therefore, the molecular motion of water is restricted in directions perpendicular to axons and preferentially diffuses in parallel directions. The most commonly used quantity of several indices obtained from DTI comprises fractional anisotropy (FA), which measures the degree of "anisotropy" in water diffusion.?

Although MS lesions are usually recognized as hyperintense on conventional T2-weighted images and hypointense on T1-weighted images, several studies have reported DTI metric abnormalities that include FA in normal-appearing WM (NAWM) around MS lesions. ${ }^{10-16}$ The FA abnormalities existing in NAWM can result from

Address correspondence to: Masaaki Niino, Department of Clinical Research, National Hospital Organization Hokkaido Medical Center, Yamanote 5-jo 7-chome, Nishi-ku, Sapporo 063-0005, Japan. Tel.: +81-11-611-8111, email: niino.masaaki.tc@mail.hosp.go.jp

Date of Submission: 7 August 2021; Date of Acceptance: 24 August 2021

https://doi.org/10.54029/2021pnk 
axonal loss causing an expanded extracellular space and gliosis, a disorganized and dense pathologic process that can reduce anisotropy. ${ }^{16,17}$ Therefore, FA is more sensitive than conventional MRI for the assessment of WM integrity in MS.

Surveying using oval regions of interest (ROIs) is not suitable for measurement of NAWM FA values as MS is a widespread disease that extends beyond the margins of focal lesions..$^{17,18}$ Therefore, histogram analysis has been used in previous reports ${ }^{11,19-23}$, showing that conventional MRI-visible MS lesions and NAWM exhibit lower FA than the WM of controls. While focal demyelinating lesions is a feature of conventional MRI findings for MS, the correlation between disability and MRI measurements - such as $\mathrm{T} 2$ lesion burden, number of new T2 lesions, enhancing lesions, and so on is relatively poor and has even been characterized as constituting a "clinical-radiological paradox". 7,24

In this study histogram analysis was used to estimate WM damage during MS by measuring WM ROIs while thoroughly eliminating gray matter (GM) and cerebrospinal fluid (CSF) contamination. The FA distribution obtained from the ROI is a similar Gaussian curve, which includes not only the mean but also variance, such as standard deviation (SD), kurtosis, and skewness of NAWM. To date, little information is available concerning the variance measurements of FA for NAWM. In this study, we investigated the most useful value for estimation of NAWM damage using the median, SD, kurtosis, and skewness of the FA histogram.

\section{METHODS}

\section{Participants}

The participants in this study were outpatients with MS, diagnosed using the 2010 revised McDonald criteria $^{25}$, from the Department of Neurology at our hospital. MS patients who had relapses within 1 month before the examination were excluded from this study. Age- and sex-matched healthy controls aged between 20 and 40 years were also included in the study. The control subjects were required to be free of any neurological and/or psychiatric disorders, such as cerebral vascular diseases, epilepsy or depression. The study was conducted with the approval of the local research ethics committee and has been performed in accordance with the ethical standards laid down in the 1964 Declaration of Helsinki and its later amendments. All participants gave their informed consent prior to their inclusion in the study.

\section{Image acquisition}

A 1.5T MRI system (Achieva, Philips Medical Systems, Best, Netherlands) with a SENSEHead-8 coil was used in this study. To obtain brain FA maps, diffusion-weighted images using a single-shot echo-planar imaging sequence were acquired using the following parameters: field of view, $224 \times 224 \mathrm{~mm}$; matrix size, $112 \times 112$; sensitivity-encoding parallel imaging factor, 2 ; repetition time, $9011 \mathrm{~ms}$; echo time, $80 \mathrm{~ms}$; fat saturation, spectral attenuated with inversion recovery; b-value, $800 \mathrm{~s} / \mathrm{mm}^{2}$; number of motion probing gradient direction, 33; number of slices, 70; slice thickness, $2 \mathrm{~mm}$; slice gaps, $0 \mathrm{~mm}$.

\section{Data processing}

FA images were calculated following bulk motion collection via the console application. Both b0 and FA images were segmented into gray matter and $\mathrm{WM}\left(\mathrm{WM}_{\mathrm{b} 0}\right.$ and $\mathrm{WM}_{\mathrm{FA}}$, respectively) using the SPM12 software package. ${ }^{26}$ The WM pixels were defined as those that applied to both $\mathrm{WM}_{\mathrm{b} 0}$ and $\mathrm{WM}_{\mathrm{FA}}$ (Figure 1). The WM ROI calculated from the $\mathrm{WM}_{\mathrm{b} 0}$ eliminated MS plaque and the $\mathrm{WM}_{\mathrm{FA}}$ represents less gray matter contamination. Therefore, the WM pixels have both of these advantages incorporated. Subsequently, the WM ROIs were carefully compared to b0 and FA images, and pixels that were likely to contain a high amount of GM, CSF or vessels were manually removed. The median, SD, kurtosis, and WM skewness of each subject were calculated by an in-house application (Matlab, R2017b, The MathWorks, Natick, Massachusetts, USA). An Expanded Disability Status Scale (EDSS) score was assessed for each patient.

\section{Statistical analysis}

A Shapiro-Wilk test was carried out to verify the data distribution normality due to a relatively small sample size. The statistical significance of differences of each value between the two groups was assessed using Student's $t$ test if the data distributions were considered as normal, otherwise a Mann-Whitney's U test was used. All tests were 2-tailed, with differences reported as significant if $p<0.05$. Analyses were performed using SPSS 18 for Windows (IBM, Chicago, USA). Pearson's correlation coefficient $(r)$ was used to study the relationship between EDSS and each value. A receiver operating characteristics (ROC) curve 


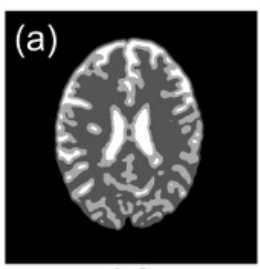

b0

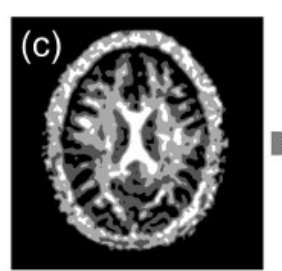

FA

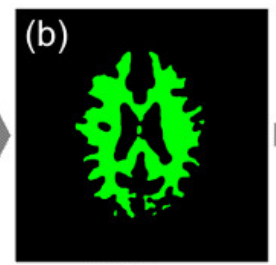

$\mathrm{WM}_{\mathrm{bO}}$

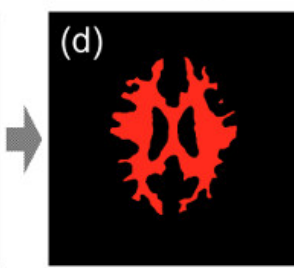

$\mathrm{WM}_{\mathrm{FA}}$

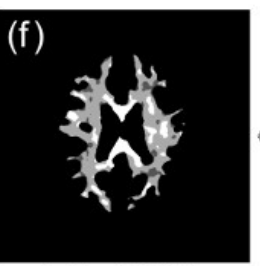

FA of WM

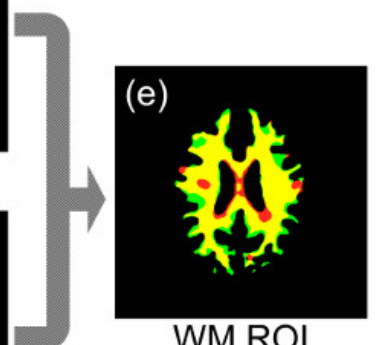

WM ROI

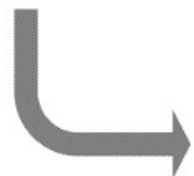

Figure 1. Fractional anisotropy (FA) extraction of white matter (WM)

The b0 maps (a) and FA maps (c) were segmented into $\mathrm{WM}\left(\mathrm{WM}_{\mathrm{b} 0}, \mathrm{WM}_{\mathrm{FA}}\right.$, respectively; b, d). Image (e) is an overlaid image of $\mathrm{WM}_{\mathrm{b} 0}$ shown in green area and $\mathrm{WM}_{\mathrm{FA}}$ shown in red area. The $\mathrm{WM}$ region of interest (ROI) was generated based on the common area (yellow color) of $\mathrm{WM}_{\mathrm{b} 0}$ and $\mathrm{WM}_{\mathrm{FA}}$ (e). The pixels not belonging to normal-appearing WM were eliminated by applying WM ROIs (f).

was generated, and the area under the curve (AUC), $p$ value, sensitivity, specificity, positive predictive value (PPV), and negative predictive value (NPV) were calculated to determine the best indices.

\section{RESULTS}

The patient group consisted of two males and six females with mean age of $34.2 \pm 6.0$ years old (Table 1). All patients were classified as having relapsing-remitting MS. Three healthy controls having an unruptured arterial aneurysm, asymptomatic cerebral infarction, or strong metal artifacts from dental crowns were excluded. One healthy control exhibiting an aneurysm discovered during the examination was excluded because the possibility that aneurysms impact SPM12 analysis could not be eliminated. Finally, mean age $( \pm$ SD) of the 12 healthy control participants (three

Table 1: Clinical profiles of the included patients

\begin{tabular}{cccccc}
\hline Case No. & Age (years)/Sex & Clinical phenotype & $\begin{array}{c}\text { Disease duration } \\
\text { (years) }\end{array}$ & EDSS & DMD \\
\hline 1 & $37 / \mathrm{F}$ & $\mathrm{RR}$ & 5 & 2.0 & natalizumab \\
2 & $36 / \mathrm{F}$ & $\mathrm{RR}$ & 11 & 4.5 & natalizumab \\
3 & $37 / \mathrm{F}$ & $\mathrm{RR}$ & 20 & 1.0 & fingolimod \\
4 & $24 / \mathrm{F}$ & $\mathrm{RR}$ & 6 & 1.5 & none \\
5 & $26 / \mathrm{M}$ & $\mathrm{RR}$ & 3 & 1.5 & fingolimod \\
6 & $43 / \mathrm{M}$ & $\mathrm{RR}$ & 1.25 & 0 & none \\
7 & $34 / \mathrm{F}$ & $\mathrm{RR}$ & 9 & 3.5 & fingolimod \\
8 & $33 / \mathrm{F}$ & $\mathrm{RR}$ & 9 & 1 & fingolimod \\
\hline
\end{tabular}

RR; relapsing-remitting, EDSS; Expanded disability status scale, DMD; disease modifying drug. 


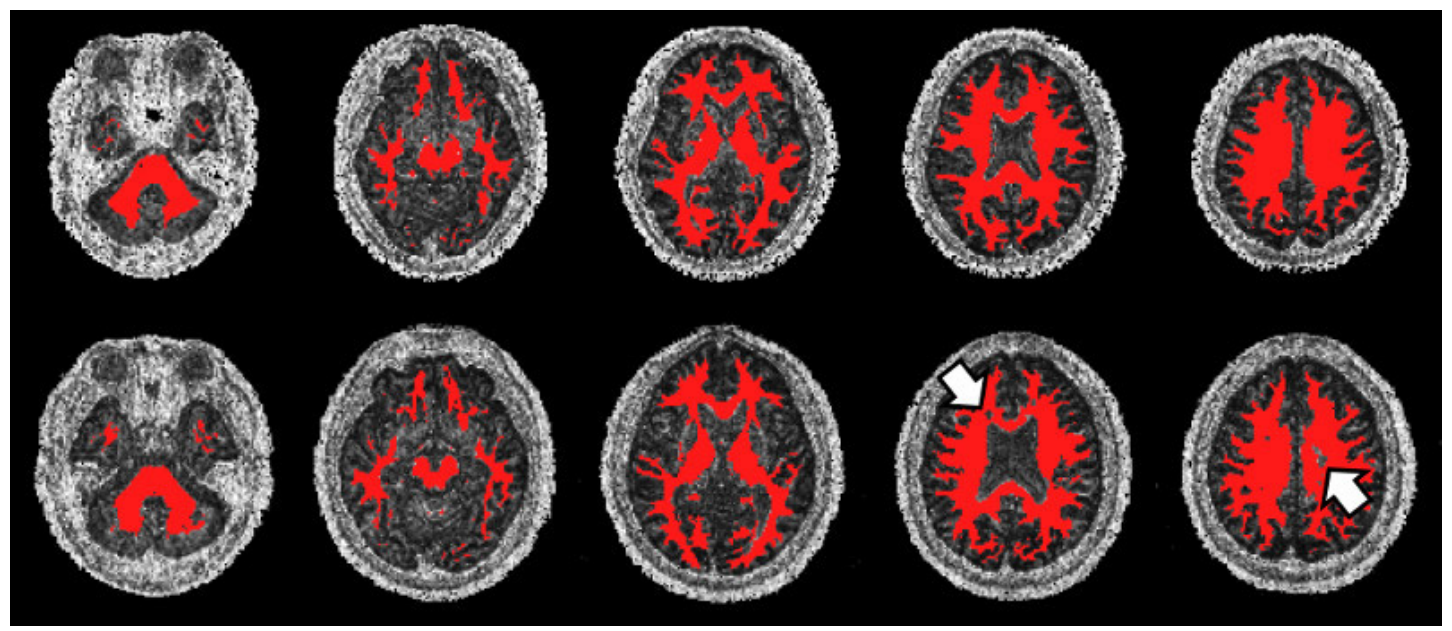

Figure 2. Typical white matter masks of a healthy subject (top) and a multiple sclerosis (MS) patient (bottom) Red pixels are superimposed on fractional anisotropy maps. MS lesions (arrow) are not included in the mask.

males and nine females) was $31.6 \pm 5.1$ years, which was not significantly different from that of the MS patient group $(p=0.31)$.

Figure 2 demonstrates the typical data of a WM mask, consisting of WM pixels of the cerebrum including corpus callosum, the medulla of the cerebellum, and the brainstem, and excluding MS lesions and gray matter of the cerebrum. The FA WM histogram of the MS patient group was compared with that of healthy controls (Figure 3). FA decrease in patients with MS was observed compared with controls and exhibited leaning toward the left side.

The distributions of the median, SD, kurtosis and skewness of each subject are shown in Figure 4. The results of the Shapiro-Wilk test showed that all indexes exhibited normal distributions. Thus, the value comparisons

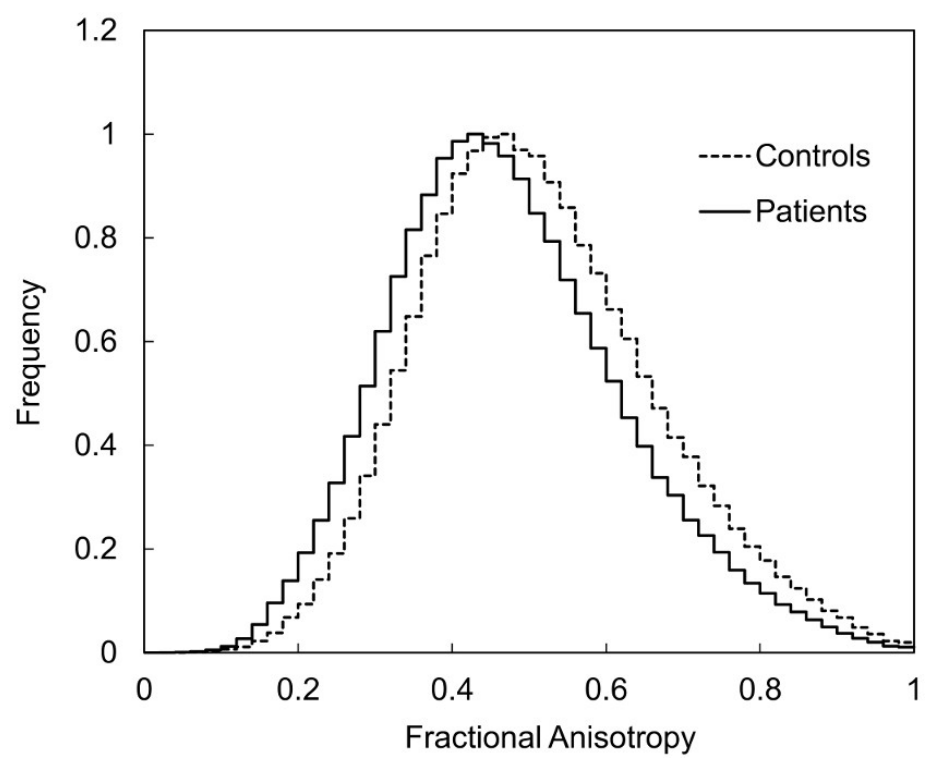

Figure 3. Normalized histograms of fractional anisotropy from normal-appearing white matter Fractional anisotropy (FA) histograms of white matter (WM) from 8 patients with MS (solid line) and 12 healthy controls (dotted line). For comparison between the groups, the frequency of FA was normalized using the peak height of histogram. Each class value was divided by the peak height of the histogram so that the vertical axis could fall within the range of 0 to 1 . In other words, the vertical axis is the ratio to the peak value. The histogram shape of patients with MS is shifted toward the left. 
$\mathbf{a}$

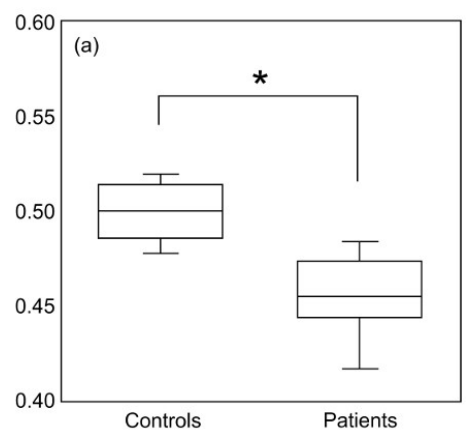

c

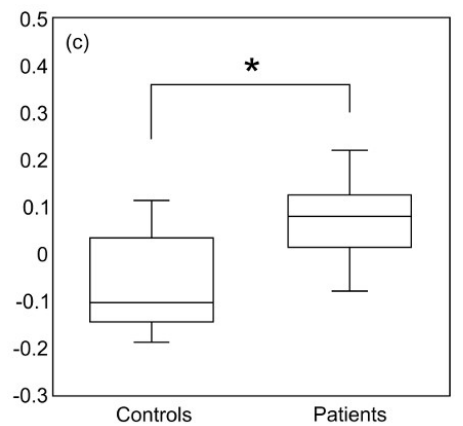

b

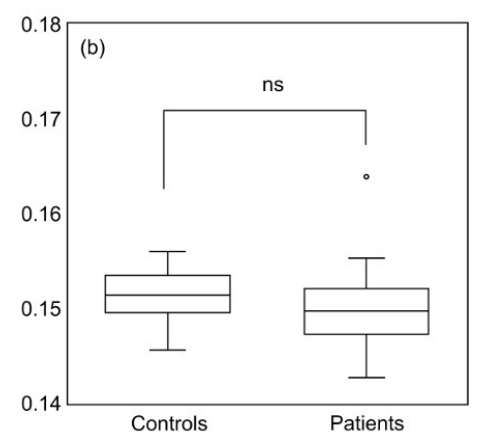

d

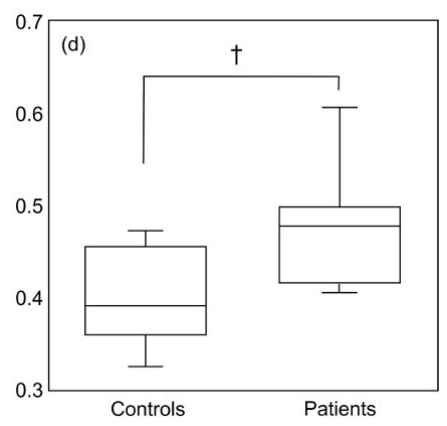

Figure 4. Boxplots showing the distributions of various indices obtained from histograms

Median (a), standard deviation (b), kurtosis (c), and skewness (d) of fractional anisotropy of white matter for each subject. The median, kurtosis and skewness show significant differences between control and patient groups as analyzed by Student's t test $(* p<0.01, \uparrow p<0.05$, ns: not significant)

between the two groups were carried out using the Student's $t$ test. While the SD of the healthy controls did not differ significantly from that of the patient group ( $p=0.83$ ), the median, kurtosis, and skewness were significantly higher in healthy controls $(p<0.0001,<0.01,<0.05$, respectively). A trend of inverse associations was observed between median and EDSS values ( $r=-0.77, p=0.027$ ); however, no significant correlations were observed between EDSS and $\mathrm{SD}$, kurtosis or skewness (Figure 5). ROC curve analysis confirmed the median as the best index to distinguish patients with MS from controls (Figure 6). Area under the curve of the median was 0.948 , and sensitivity and specificity of the median were $87.5 \%$ and $83.3 \%$ (cutoff value $=$ 0.482 ). Other detailed ROC results are shown in Table 2 .

\section{DISCUSSION}

Previous studies reported that the FA peak position of NAWM obtained from histogram differed between patients with MS and heathy controls. ${ }^{11,19,21}$ The peak position was used in those studies because the ROI includes multiple tissue components, such as GM, WM and MS plaque, and the average obtained from an entire tissue may not represent each of its features. The ROIs primarily consist of WM and GM components, and the peak histogram position is more influenced by the GM components, which displays a higher peak than WM. In this study, we removed the GM and CSF components as well as large vessels from the ROI, because WM is considered to be the primary pathological lesions in MS. Taken together, we focused on the WM of MS in this study and measured the median, SD, kurtosis, and skewness of FA of WM.

The results of the present study indicate that the FA median is superior to SD, kurtosis, skewness for estimation of WM damage in patients with MS. There exists a clear distinction between the median and other indices. The value of the median indicates the average FA value of NAWM, and SD, kurtosis, skewness indicates the FA distribution. The FA distribution in WM of patients with MS shows roughly two peaks, one representing the NAWM value and the other MS lesions. However, the WM ROI excluded MS lesions in this study. Therefore, lesion burden does not easily impact the median. On the other hand, if the spread of WM damage is paralleled with the worsening of 
a

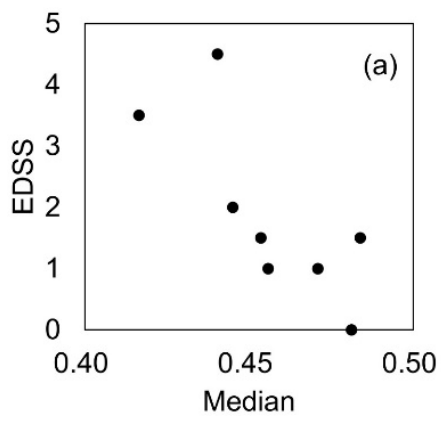

c

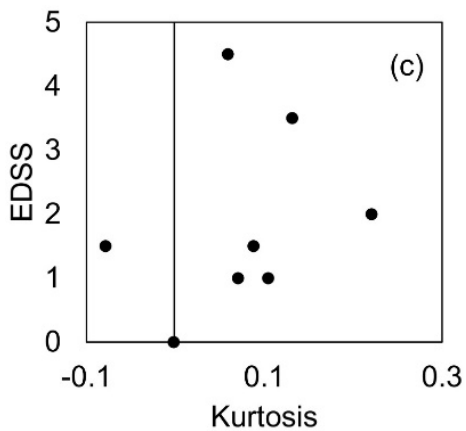

b

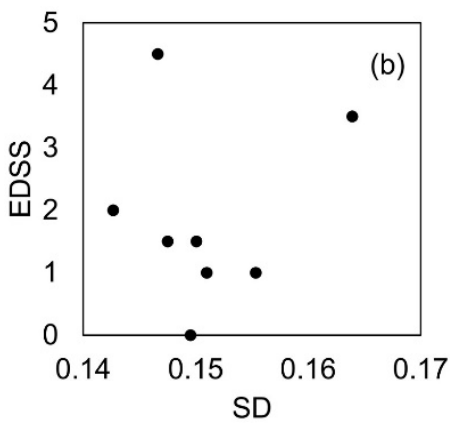

d

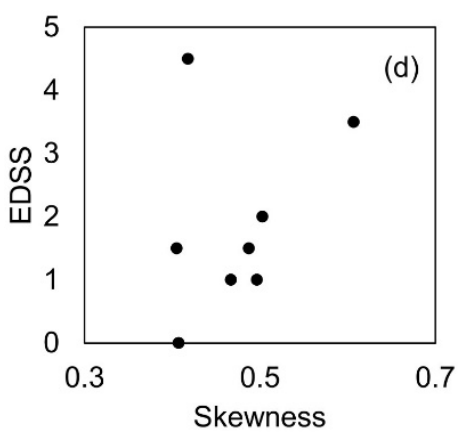

Figure 5. Associations between the median, standard deviation (SD), kurtosis and skewness of fractional anisotropy for normal-appearing white matter and Expanded Disability Status Scale (EDSS)

The scatterplots of relationships between the median (a), SD (b), kurtosis (c) or skewness (d) of fractional anisotropy and EDSS are shown. A trend of inverse associations was observed between median and EDSS $(r=-0.77, p=0.027$ ), whereas no significant correlations were found between EDSS and SD, kurtosis or skewness. This suggests that myelin damage increases as multiple sclerosis-related disability progresses.

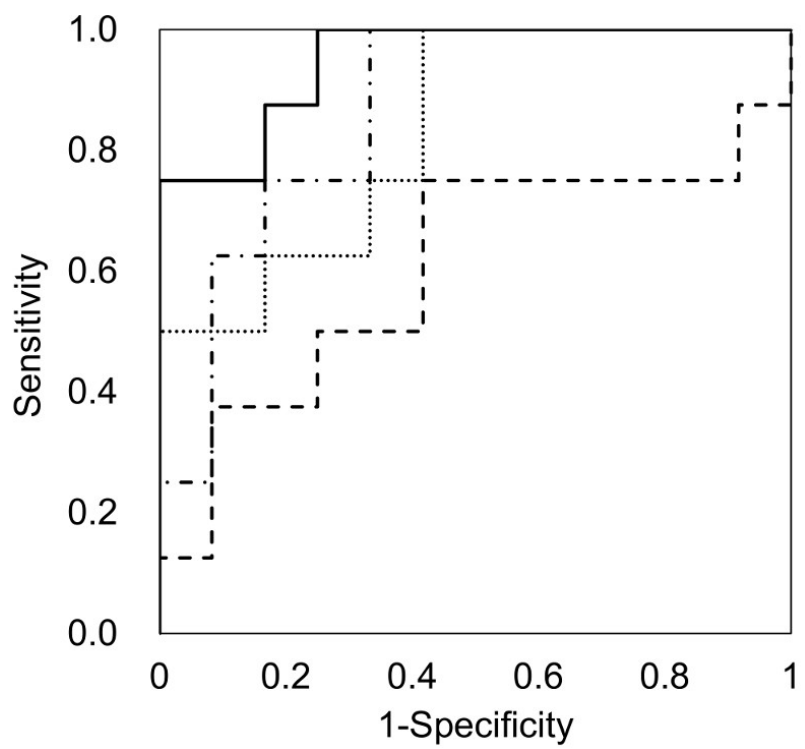

Figure 6. Receiver operating characteristics curve generated to determine optimal indices of the FA histogram A receiver operating characteristics curve was plotted to compare the differentiation performance of median (solid line), standard deviation (dashed line), kurtosis (dash dot line), and skewness (dotted line). The optimal index was the median (area under curve; 0.948). 
Table 2: Results of receiver operating characteristics curve

\begin{tabular}{cccccccc}
\hline & AUC & Cutoff value & P value & $\begin{array}{c}\text { Sensitivity } \\
(\boldsymbol{\%})\end{array}$ & $\begin{array}{c}\text { Specificity } \\
(\boldsymbol{\%})\end{array}$ & $\begin{array}{c}\text { PPV } \\
(\boldsymbol{\%})\end{array}$ & $\begin{array}{c}\text { NPV } \\
(\boldsymbol{\%})\end{array}$ \\
\hline Median & 0.948 & 0.482 & $<0.001$ & 87.5 & 83.3 & 77.8 & 90.9 \\
SD & 0.604 & 0.151 & 0.440 & 75.0 & 58.3 & 54.5 & 77.8 \\
Kurtosis & 0.865 & 0.052 & $<0.01$ & 75.0 & 83.3 & 75.0 & 83.3 \\
Skewness & 0.833 & 0.417 & $<0.05$ & 75.0 & 66.7 & 60.0 & 80.3 \\
\hline
\end{tabular}

AUC; area under the curve, PPV; positive predictive value, NPV; negative predictive value, SD; standard deviation

MS symptoms, FA may decrease in a wide area, thereby resulting in a decrease in the median. The results of this study indicate that only the median is correlated with EDSS, and supports this theory. Although the distribution of FA values in WM is consistent with a normal distribution, the median value, instead of the mean value, was used in this analysis because low spatial resolution in FA images might cause contamination of other tissues than WM.

Regardless of the shape of the histogram, the FA of NAWM appears to be lower than that of healthy controls. FA reflects the amount of myelin; ${ }^{27-29}$ therefore, the decrease of FA correlates with the water diffusivity and isotropy elevation caused by extracellular space expansion..$^{30,31}$ However, the relationship between FA and EDSS differs in the literature. ${ }^{11,15,16,19,23,32}$ FA shows high reproducibility ${ }^{11}$ and reflects pathological changes.$^{16}$ However, Cercignani and colleagues pointed out the many limitations of EDSS as well as the role of spinal cord damage in determining neurologic disability. ${ }^{19}$ This may be a contributing factor to this variability, and the number of cases in many of the aforementioned studies was small, so it can be considered that these data are interpreted arbitrarily. This is similar to our result concerning the relationship between FA and EDSS.

Although the SD did not differ significantly between the patient and control groups, there were statistically significant differences between kurtosis and skewness. The kurtosis of the patient group may have been higher than that of the healthy control group because WM containing various structures was homogenized due to the presence of MS. In addition, skewness of the patient group is higher than that of the healthy group likely due to the FA distribution of the patient group shifting to be lower than that of healthy controls, indicating a decline in myelin content. Both indices overlap abundantly between the two groups, thus the distinction made by the median is optimal for discrimination between the groups. Both kurtosis and skewness are functions of an average, and SD did not differ between controls and patients with MS, thus they are influenced by differences in FA median, not SD. Only the median showed a correlation with EDSS. In summary, the FA median is more important for assessment of MS symptoms than distribution. The ROC analysis results indicate that the FA median is the most accurate indicator to distinguish patients with MS from healthy controls. In addition, although we do not show data concerning the average of FA, the same tendency existed for these values as for the median.

One of the limitations of this study is that we could not estimate whether the change of FA was only due to the effect of MS. It is known that FA change is also associated with MR field strength ${ }^{33}$, aging ${ }^{34-36}$, post-traumatic stress disorder $^{37}$, schizophrenia ${ }^{38}$ and other mental disorders $^{39,40}$, human immunodeficiency virus infection ${ }^{41}$, moyamoya disease ${ }^{42}$, and Parkinson's disease. ${ }^{43-45}$ Therefore, the patient's background should be considered in the evaluation of FA. However, discrimination capability of FA is superior for comparison based on nonspecific WM changes caused by collagen disease, vasculitis, and chronic infarction in study of typical MS WM and is of great interest for study in the future. As discussed above, aging could affect FA change. ${ }^{34-36}$ In our study, there was some difference between the mean ages of the patient and control groups, although that was not different statistically $(p=0.31)$. However, the difference was only about 3 years between the two groups. Kochunov et al. demonstrated that FA decreases 0.01 in 10 years $^{34}$, which was about $7 \%$ of SD of FA distribution. Therefore, we considered that the difference of ages between the two groups did not affect the result of our study significantly. Another limitation of this study is the small number of participants. However, the statistical power of median, kurtosis and skewness were 0.8 or higher, although that of SD was 0.064. These data could support the significance of our study. However, future studies with more participants 
would improve the accuracy of the relationship and ROC analyses.

There are some reports indicating that certain MRI methods - like diffusion kurtosis imaging and q-space imaging - are more optimal than FA. ${ }^{46,47}$ However, these methods require a special imaging acquisition sequence and data processing . In contrast, the FA has the advantage in that it does not require any additional measurement equipment and can be calculated at any MRI facility.

We determined the FA histogram of WM with high accuracy, and examined whether differences in median and distribution could distinguish patients with MS from healthy controls and assess the severity of symptoms. As a result, we clarify that the median value of FA can distinguish between patients with MS and healthy controls with high accuracy.

\section{DISCLOSURE}

Financial support: This study was supported, in part, by a Health and Labour Sciences Research Grant on Rare and Intractable Diseases (Validation of Evidence-based Diagnosis and Guidelines, and Impact on QOL in Patients with Neuroimmunological Diseases) from the Ministry of Health, Labour and Welfare of Japan.

\section{Conflict of interest: None}

\section{REFERENCES}

1. Noseworthy JH, Lucchinetti C, Rodriguez M, Weinshenker BG. Multiple sclerosis. $N$ Engl J Med 2000;343(13):938-52.

2. Compston A, Coles A. Multiple sclerosis. Lancet 2002;359(9313):1221-31.

3. McFarlin DE, McFarland HF. Multiple sclerosis (first of two parts). N Engl J Med 1982;307(19):1183-8.

4. Lladó X, Oliver A, Cabezas M, et al. Segmentation of multiple sclerosis lesions in brain MRI: a review of automated approaches. Inf Sci 2012;186(1):164-85.

5. Gebarski SS, Gabrielsen TO, Gilman S, Knake JE, Latack JT, Aisen AM. The initial diagnosis of multiple sclerosis: clinical impact of magnetic resonance imaging. Ann Neurol 1985;17(5):469-74.

6. Kirshner HS, Tsai SI, Runge VM, Price AC. Magnetic resonance imaging and other techniques in the diagnosis of multiple sclerosis. Arch Neurol 1985;42(9):859-63.

7. Goodin DS. Magnetic resonance imaging as a surrogate outcome measure of disability in multiple sclerosis: Have we been overly harsh in our assessment? Ann Neurol 2006;59(4):597-605.

8. Basser PJ, Mattiello J, LeBihan D. Estimation of the effective self-diffusion tensor from the NMR spin echo. J Magn Reson B 1994;103(3):247-54.

9. Basser PJ, Pierpaoli C. Microstructural and physiological features of tissues elucidated by quantitative-diffusion-tensor MRI. J Magn Reson B 1996;111(3):209-19.

10. Lipp I, Jones DK, Bells S, et al. Comparing MRI metrics to quantify white matter microstructural damage in multiple sclerosis. Hum Brain Mapp 2019;40(10):2917-32.

11. Cassol E, Ranjeva JP, Ibarrola D, et al. Diffusion tensor imaging in multiple sclerosis: a tool for monitoring changes in normal-appearing white matter. Mult Scler 2004;10(2):188-96.

12. Castriota-Scanderbeg A, Fasano F, Hagberg G, Nocentini U, Filippi M, Caltagirone C. Coefficient Dav is more sensitive than fractional anisotropy in monitoring progression of irreversible tissue damage in focal nonactive multiple sclerosis lesions. AJNR Am J Neuroradiol 2003;24(4):663-70.

13. Bammer R, Augustin M, Strasser-Fuchs S, et al. Magnetic resonance diffusion tensor imaging for characterizing diffuse and focal white matter abnormalities in multiple sclerosis. Magn Reson Med 2000;44(4):583-91.

14. Werring DJ, Clark CA, Barker GJ, Thompson AJ, Miller DH. Diffusion tensor imaging of lesions and normal-appearing white matter in multiple sclerosis. Neurology 1999;52(8):1626-32.

15. Filippi M, Cercignani M, Inglese M, Horsfield MA, Comi G. Diffusion tensor magnetic resonance imaging in multiple sclerosis. Neurology 2001;56(3):304-11.

16. Ciccarelli O, Werring DJ, Wheeler-Kingshott CAM, et al. Investigation of MS normal-appearing brain using diffusion tensor MRI with clinical correlations. Neurology 2001;56(7):926-33.

17. Allen IV, McQuaid S, Mirakhur M, Nevin G. Pathological abnormalities in the normal-appearing white matter in multiple sclerosis. Neurol Sci 2001;22(2):141-4.

18. Adams CWM. Pathology of multiple sclerosis: progression of the lesion. Br Med Bull 1977;33(1):1520.

19. Cercignani M, Inglese M, Pagani E, Comi G, Filippi M. Mean diffusivity and fractional anisotropy histograms of patients with multiple sclerosis. AJNR Am J Neuroradiol 2001;22(5):952-8.

20. Rovaris M, Bozzali M, Iannucci G, et al. Assessment of normal-appearing white and gray matter in patients with primary progressive multiple sclerosis. Arch Neurol 2002;59(9):1406-12.

21. Rashid W, Hadjiprocopis A, Griffin CM, et al. Diffusion tensor imaging of early relapsing;/remitting multiple sclerosis with histogram analysis using automated segmentation and brain volume correction. Mult Scler 2004;10(1):9-15.

22. Gallo A, Rovaris M, Riva R, et al. Diffusion-tensor magnetic resonance imaging detects normalappearing white matter damage unrelated to shortterm disease activity in patients at the earliest clinical stage of multiple sclerosis. Arch Neurol 2005;62(5):803-8.

23. Iannucci G, Rovaris M, Giacomotti L, Comi G, Filippi M.Correlation of multiple sclerosis measures derived from T2-weighted, T1-weighted, magnetization transfer, and diffusion tensor MR imaging. AJNR 
Am J Neuroradiol 2001;22(8):1462-7.

24. Barkhof F. The clinico-radiological paradox in multiple sclerosis revisited. Curr Opin Neurol 2002;15(3):239-45.

25. Polman $\mathrm{CH}$, Reingold $\mathrm{SC}$, Banwell $\mathrm{B}$, et al. Diagnostic criteria for multiple sclerosis: 2010 revisions to the McDonald criteria. Ann Neurol 2011;69(2):292-302.

26. http://www.fil.ion.ucl.ac.uk/spm/software/spm12/.

27. Schmierer K, Wheeler-Kingshott CAM, Boulby PA, et $a l$. Diffusion tensor imaging of post mortem multiple sclerosis brain. Neurolmage 2007;35(2):467-77.

28. Moll NM, Rietsch AM, Thomas S, et al. Multiple sclerosis normal-appearing white matter: pathologyimaging correlations. Ann Neurol 2011;70(5):764-73.

29. Chang EH, Argyelan M, Aggarwal M, et al. Diffusion tensor imaging measures of white matter compared to myelin basic protein immunofluorescence in tissue cleared intact brains. Data Brief 2016;10:438-43.

30. Tievsky AL, Ptak T, Farkas J. Investivation of apparent diffusion coefficient and diffusion tensor anisotropy in acute and chronic multiple sclerosis lesions. AJNR Am J Neuroradiol 1999;20(8):1491-9.

31. Graulières E, Lotterie JA, Cassol E, Gerdelat A, Clanet M, Berry I. Relevance of the skewness index in DTI exploration of multiple sclerosis. Magn Reson Mater Phy 2009;22(2):89-100.

32. Herbert E, Engel-Hills P, Hattingh C, et al. Fractional anisotropy of white matter, disability and blood iron parameters in multiple sclerosis. Metab Brain Dis 2018;33(2):545-57.

33. Fushimi Y, Miki Y, Okada T, et al. Fractional anisotropy and mean diffusivity: comparison between 3.0-T and 1.5-T diffusion tensor imaging with parallel imaging using histogram and resion of interest analysis. NMR Biomed 2007;20(8):743-8.

34. Kochunov P, Thompson PM, Lancaster JL, et al. Relationship between white matter fractional anisotropy and other indices of cerebral health in normal aging: Tract-based spatial statistics study of aging. NeuroImage 2007;35(2):478-87.

35. Abe $\mathrm{O}$, Aoki $\mathrm{S}$, Hayashi $\mathrm{N}$, et al. Normal aging in the central nervous system: quantitative MR diffusiontensor analysis. Neurobiol Agin 2002;23(3):433-41.

36. Furutani K, Harada M, Minato M, Morita N, Nishitani H. Regional changes of fractional anisotropy with normal aging using statistical parametric mapping (SPM). J Med Invest 2005;52(3-4):186-90.

37. Zhang L, Zhang Y, Li L, et al. Different white matter abnormalities between the first-episode, treatmentnaive patients with posttraumatic stress disorder and generalized anxiety disorder without comorbid conditions. J Affect Disord 2011;133(1-2):294-9.

38. Burns J, Job D, Bastin ME, et al. Structural disconnectivity in schizophrenia: a diffusion tensor magnetic resonance imaging study. Br J Psychiatry 2003;182:439-43.

39. Lochner C, Fouché JP, du Plessis S, et al. Evidence for fractional anisotropy and mean diffusivity white matter abnormalities in the internal capsule and cingulum in patients with obsessive-compulsive disorder. J Psychiatry Neurosci 2012;37(3):193-9.

40. Bozzali M, Falini A, Franceschi M, et al. White matter damage in Alzheimer's disease assessed in vivo using diffusion tensor magnetic resonance imaging. J Neurol Neurosurg Psychiatry 2002;72(6):742-6.

41. Ragin AB, Storey P, Cohen BA, Epstein LG, Edelman RR. Whole brain diffusion tensor imaging in HIV-associated cognitive impairment. AJNR Am J Neuroradiol 2004;25(2):195-200.

42. Mori N, Miki Y, Fushimi Y, et al, Cerebral infarction associated with moyamoya disease: histogrambased quantitative analysis of diffusion tensor imaging -a preliminary study. Magn Reson Imaging 2008;26(6):835-40.

43. Kamagata K, Motoi Y, Tomiyama $\mathrm{H}$, et al. Relationship between cognitive impairment and white-matter alteration in Parkinson's disease with dementia: tract-based spatial statistics and tractspecific analysis disease with dementia: tract-based spatial statistics and tract-specific analysis. Eur Radiol 2013;23(7):1946-55.

44. Skidmore FM, Spetsieris PG, Anthony T, et al. A full-brain, bootstrapped analysis of diffusion tensor imaging robustly differentiates Parkinson disease from healthy controls. Neuroinformatics 2015;13(1):7-18.

45. Matsui H, Nishinaka K, Oda M, Niikawa H, Kubori T, Udaka F. Dementia in Parkinson's disease: diffusion tensor imaging. Acta Neurol Scand 2007;116(3):17781.

46. Assaf Y, Chapman J, Ben-Bashat D, et al. White matter changes in multiple sclerosis: correlation of q-space diffusion MRI and 1H MRS. Magn Reson Imagin 2005;23(6):703-10.

47. Hori M, Fukunaga I, Masutani Y, et al. Visualizing non-gaussian diffusion: clinical application of q-space imaging and diffusional kurtosis imaging of the brain and spine. Magn Reson Med Sci 2012;11(4):221-33. 